\title{
Effect of Growth Hormone and Calorie Restriction on the Ex- pression of Antioxidant Enzymes in the Liver and Kidney of Growth Hormone Receptor Knockout Mice
}

\author{
Khalid A Al-Regaiey
}

Department of Physiology, College of Medicine, King Saud University, Riyadh 11461, Kingdom of Saudi Arabia.

Received: March 7, 2012 / Accepted: October 20, 2012

\begin{abstract}
Caloric restriction (CR) can delay aging and prolong life span and these actions may be related to reduced oxidative damage. Mice with disrupted growth hormone (GH) receptor/ binding protein knockout (GHRKO) live significantly longer than their normal siblings. Therefore, it is of interest to examine the effects of chronic CR on hepatic and renal antioxidant enzymes as well as lipid peroxidation (LP) as an oxidative stress marker in GHRKO mice. Female GHRKO and normal mice were either fed ad libitum (AL) or subjected to $30 \% \mathrm{CR}$ starting at 2 months of age and examined at the age of 9 months. In the liver, catalase (CAT) activity was significantly increased in GHRKO-AL as compared to normal control -AL animals. CR reduced CAT activity in both GHRKO and normal phenotypes. $\mathrm{Cu} / \mathrm{Zn}$ superoxide dismutase (SOD1) activity was also higher in GHRKO-AL as compared to normalAL mice. However, CR reduced SOD1 activity in GHRKO mutants. Glutathione peroxidase (GPx) activity was significantly decreased in GHRKO-AL mice and further reduced in GHRKO-CR group of animals. CR significantly increased LP in GHRKOs while its activity was not altered in GHRKOAL group of mice. In the kidney, CAT activity was lower in GHRKO-AL as compared to normal-AL, however CR did not induce any significant effect in both phenotypes. Similarly, SOD1 levels were significantly lower in GHRKO than in normal mice. GPx expression was higher in GHRKO-AL as compared to control-AL. CR reduced GPx activity in GHRKO mice but increased it in controls as compared to their AL counterparts. There was no difference in LP expression between
\end{abstract}

\footnotetext{
* Corresponding author: kalregai@gmail.com
}

GHRKO-AL and normal-AL mice. However, CR significantly increased its levels in both phenotypes. Although these findings do not support the hypothesis that CR would increase the capacity of ROS defense mechanisms in GHRKO mice by increasing antioxidant enzymes levels, they do agree with some of the reported effects of $C R$ on their expression. We suspect that $\mathrm{GH}$ resistance and $\mathrm{CR}$ may affect aging by different mechanisms and if CR delays aging in GHRKO animals it is not due to changes in the activity of antioxidant enzymes.

\section{Introduction}

It is well known that caloric restriction (CR) profoundly affects physiological and pathophysiological modifications induced by aging and markedly increases life span in in a variety of species, including rats, mice, dogs, fish, flies, worms, and yeast (Weindruch and Sohal, 1997). Studies from centenarians and individuals who self-impose CR have documented beneficial effects in terms of reducing cardiovascular and diabetes-related illnesses. A research program named CALERIE (Comprehensive Assessment of the Long-term effects of Reducing Intake of Energy) had shown positive effects of $C R$ on resting metabolic rate, reducing coronary heart disease risk factors, improves insulin sensitivity, and cognitive function (Heilbronn, et al., 2006; Fontana et al., 2007; Larson-Meyer et al., 2006; Redman et al., 2007; Martin et al., 2007).

$\mathrm{CR}$ also has beneficial effect on cognitive performance in 
humans, mediated by increased insulin sensitivity and reduced inflammatory activity, leading to higher synaptic plasticity and stimulation of neurofacilitatory pathways in the brain (Witte et al (2009). In animals, CR delay the occurrence of age-associated disease such as diabetes, cancer and cardiovascular disease and enhanc insulin sensitivity (Colman et al., 2009; Wang et al., 2009).

However, the biological mechanism by which caloric restriction may exert its antiaging action is not yet understood. Mechanisms that are thought to contribute to $C R$ effects include retardation of growth, reduction of body fat, reduced insulin and glucose levels, reduced reproductive capacities, delayed neuroendocrine changes, improved DNA repair capacities, reduced body temperature and metabolic rate, amelioration of oxidative stress damage, and hormesis (Sohal and Weindruch, 1996; Mattison et al., 1998; Masoro, 1998).

The free radical theory of aging proposes that oxidative stress is a causal factor in aging process. The imbalance between free radical production and cellular antioxidant defense leads to the accumulation of oxidative damage to the cellular macromolecules. Cellular endogenous antioxidant defense includes superoxide dismutase (SOD), catalase (CAT), glutathione peroxidase (GPx), and others. The importance of antioxidant enzymes in defying oxidative stress is evidenced by the observation that overexpression of antioxidant enzymes extends lifespan in Drosophila (Orr and Sohal, 1994; Parks et al., 1998) and that treatment of $C$. elegans with synthetic SOD/CAT mimetics extended its lifespan (Melov et al., 2006).

Studies in Drosophila, C. elegans, and mutant mice provide evidence that insulin-like signaling contributes to the aging process. Mutations in the daf-2 insulin receptor-like gene or the downstream age-1 gene extend the life span of C. elegans two- to threefold (Kenyon et al 1993). Ames and Snell dwarf mice, deficient in GH (growth hormone), PRL (prolactin) and TSH (thyroid stimulating hormone), live much longer than their normal siblings (Brown-Borg et al., 1996; Flurkey et al., 2001). CR was shown to cause significant extension of lifespan in long-lived Ames dwarf mice (Bartke et al., 1999) and in long-lived daf-2 mutant C. elegans (Lakowski and Hekimi, 1998). Moreover, The

Figure 1. Weekly body weight changes for female normal and GHR-KO mice that were fed AL or subjected to $30 \%$ CR for 9 months starting at 2 months of age.

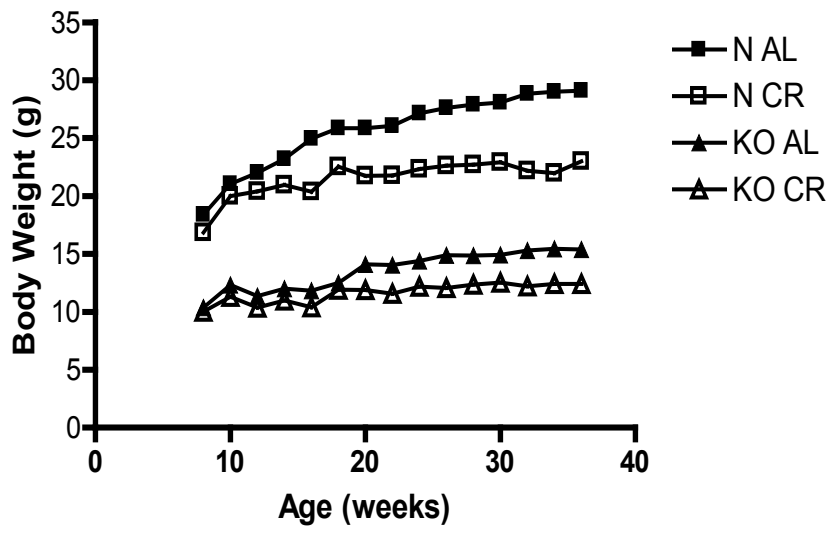

activity of two important antioxidant enzymes, CAT and Cu/ ZnSOD in the liver, kidney, and hypothalamus of Ames dwarf mice is higher than that in their normal siblings (Brown-Borg et al., 1999; Hauck and Bartke, 2000).

$\mathrm{GH}$ receptor/GH binding protein knockout mice (GHRKO) are GH-resistant and IGF-1 deficient and were also reported to live significantly longer than their normal counterparts (Tatar et al., 2003). Studies conducted in our lab had shown that in GHRKO mice, CR did not increase the overall, median or average life span of animals and it was suggested that somatotropic signaling played a key role in mediating the effects of $C R$ to enhance longevity (Bonkowski et al., 2006). In the current study we examined the effects of $C R$ on antioxidant enzymes, namely catalase (CAT), superoxide dismutase (SOD1) and glutathione peroxidase (GPX 1 ) activity and on lipid peroxidation (LP) as an oxidative damage marker in livers and kidneys of these GHRKO mice.

\section{Materials and Methods}

\section{Animals}

GHRKO female mice and normal $(N)$ littermate controls were produced in a closed colony derived from animals provided by Dr. J. Kopchick and maintained at Southern Illinois University by mating knockout $(-/-)$ males with heterozygous (+/ - ) female carriers of the disrupted $\mathrm{GHR} / \mathrm{GH}$-binding protein gene. Animals were housed on a 12-h light, 12-h dark cycle at $22 \pm 2$ ${ }^{\circ} \mathrm{C}$ and were fed (Lab Diet Formula 5008, Ralston Purina Corp., St. Louis, MO) and watered ad libitum, except as noted below. All animal procedures were approved by the laboratory animal care and user committee at Southern Illinois University School of Medicine. GHRKO mice and $\mathrm{N}$ siblings were fed either ad libitum (AL) or submitted to $30 \%$ CR protocol (eight animals per phenotype per diet) as described previously (Bonkowski et al., 2006).

\section{Samples}

At 9 months of age, animals were anesthetized by isoflurane, bled by cardiac puncture, and decapitated. To avoid stressing animals, the time between taking the animal from the cage, anesthetizing, bleeding, and decapitation was minimized $(\approx 1$ $\mathrm{min})$. Livers and kidneys were rapidly removed, quickly frozen on dry ice, and stored at $-80^{\circ} \mathrm{C}$ until processed.

Trunk blood was collected in tubes containing EDTA. After collection, blood was centrifuged at $6000 \times \mathrm{g}$ for $15 \mathrm{~min}$ at $4^{\circ} \mathrm{C}$, the plasma was collected and stored at $-80^{\circ} \mathrm{C}$.

Total proteins were obtained from whole tissue homogenates. Approximately $100 \mathrm{mg}$ liver/kidney samples were homogenized in $500 \mu \mathrm{l}$ ice-cold homogenizing buffer [20 mM Tris ( $\mathrm{pH} 7.5)$, $150 \mathrm{mM} \mathrm{NaCl}, 1 \%$ Triton 100, with protease inhibitors cocktail and phosphatase inhibitors cocktails (Sigma-Aldrich Corp.)] and spun at 14,000 $\mathrm{xg}$ for $45 \mathrm{~min}$. The supernatant was removed and stored at $-80^{\circ} \mathrm{C}$. Protein concentrations were determined using the bicinchoninic acid assay (Pierce Corp., Rockford, IL) according to the manufacturer's instructions. 
Glucose concentrations were determined using a OneTouch Ultra glucose meter (LifeScan, Milpitas, CA). Insulin was assessed by ELISA (Linco Research, Inc. St. Charles, MO), and corticosterone was determined using RIA kits from ICN.

\section{Catalase, Cu/Zn SOD, and GPx Activity}

Catalase activity was determined by UV method (Spectronic Genesys 5 spectrophotometer: Milton Roy, Rochester, NY, USA), as previously described (Aebi, 1974). One unit of the enzyme was defined as $\mu \mathrm{mol} \mathrm{H}_{2} \mathrm{O}_{2}$ disproportionated $/ \mathrm{min} / \mathrm{mg}$ protein. Total Cu/Zn SOD activity was determined by adaptation of the method described by Sun et al., (1994). One arbitrary unit (au) of SOD activity was defined as the amount of cellular protein causing $50 \%$ inhibition of the rate of nitroblue tetrazolium reduction. Catalytic activity of the samples was determined by comparison to a standard curve of commercial Cu/Zn SOD (Sigma, St. Louis, MO, USA).

GPx 1 activity was determined as described by (Tappel, 1978 ). One unit of activity was defined as the amount of enzyme required to oxidize $1 \mu \mathrm{mol}$ of reduced nicotinamide adenine dinucleotide phosphate to NADP per minute per milligram of protein.

Cellular Thiobarbituric acid-reactive substances (TBARS) were measured (as reaction equivalent to malondialdehyde) as described by Hashimoto et al. (1999). TBARS formation was expressed as nanomoles of MDA per milligram of protein.

\section{Statistical Analysis}

Data are expressed as the mean \pm SE. The statistical evaluation was performed using two-factor ANOVA (phenotype and diet), followed by Fisher's protected least significant difference test as apost hoc test. A t test was also used to evaluate the effect of diet within phenotypes and phenotype within diet.P $<0.05$ was considered significant. All statistical analyses were performed using StatView 5.0 software (SAS Institute, Inc., Cary, NC).

\section{Results and Discussion}

Despite the fact that the effects of CR and insulin/insulin-like signaling disruption share many similarities, there is evidence that they have their distinct actions. It was previously reported
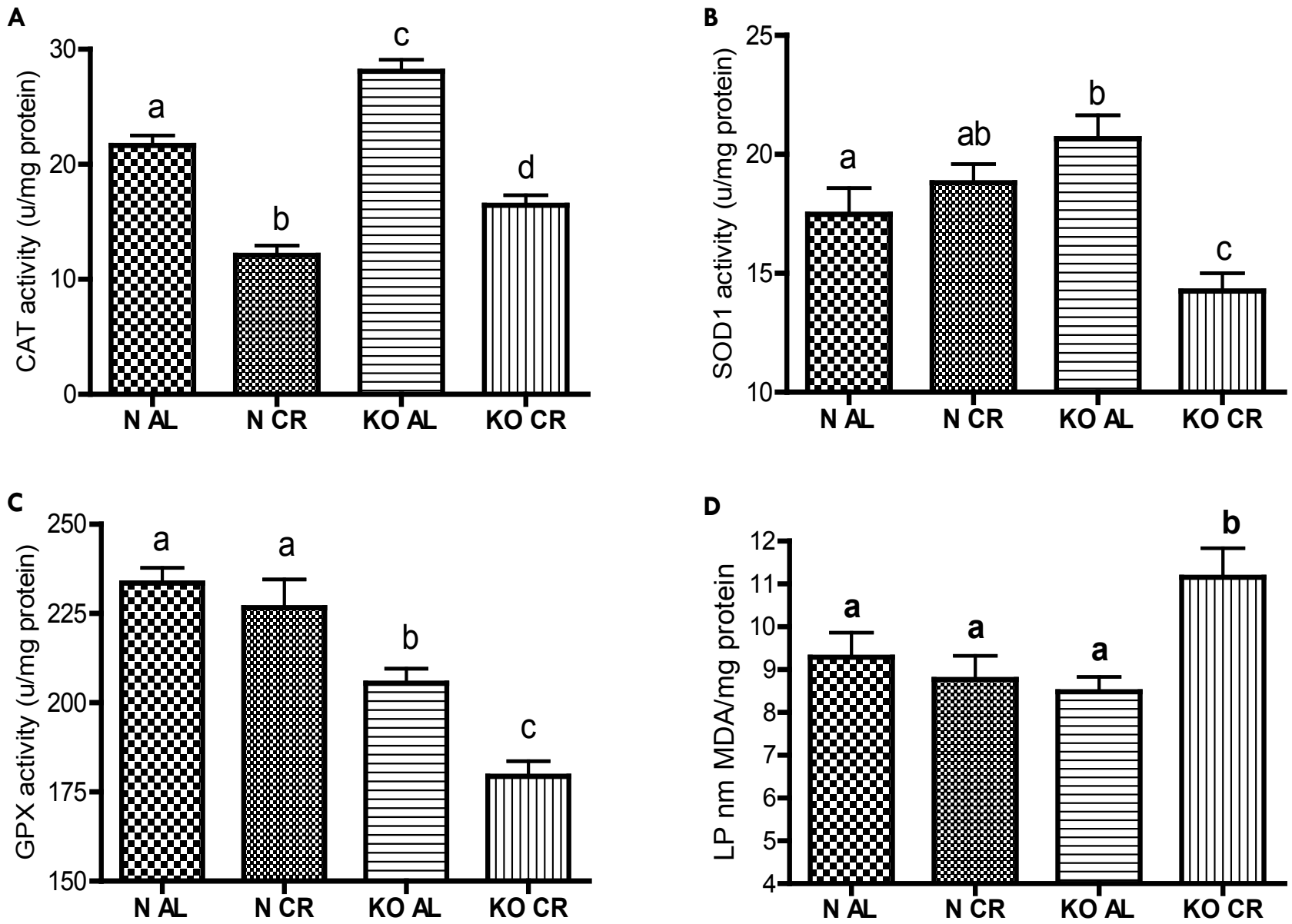

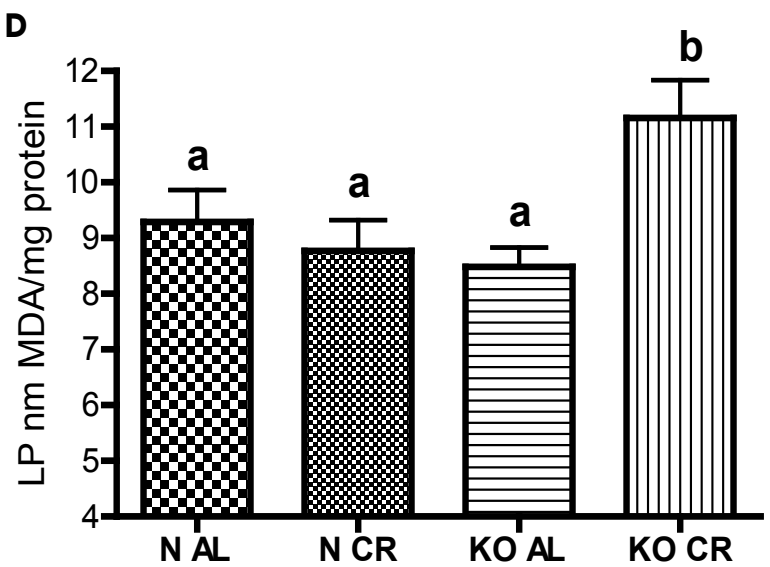

Figure 2. Hepatic antioxidant enzyme activity (A) catalase, (B) Cu/ZnSOD, (C) Gpx 1, and (D) lipid peroxidation (LP) in female GHR-KO (KO) and normal (N) mice subjected to caloric restriction (CR) or fed ad libitum (AL). Data reported as mean \pm SE. Groups that do not share a common superscript are significantly different $(p<0.05)$. 
A

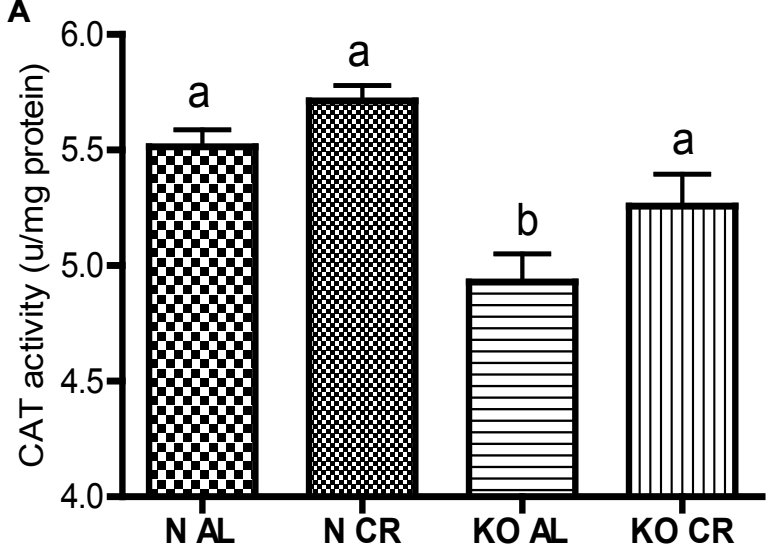

C

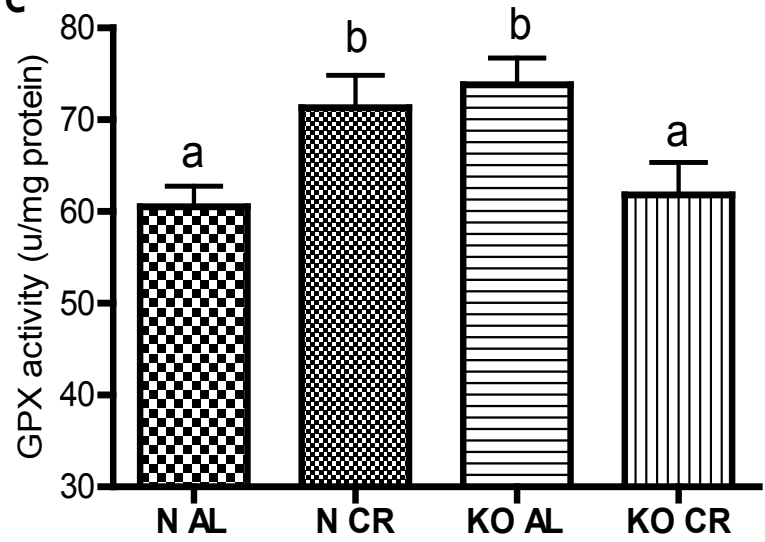

B

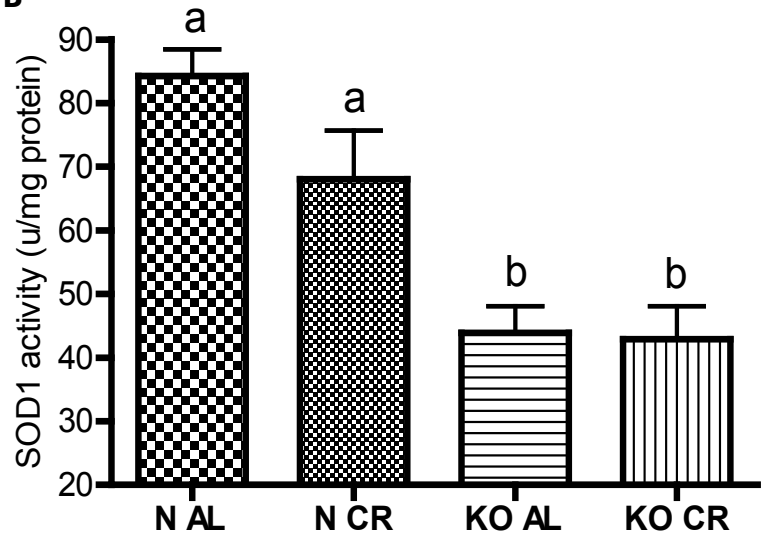

D

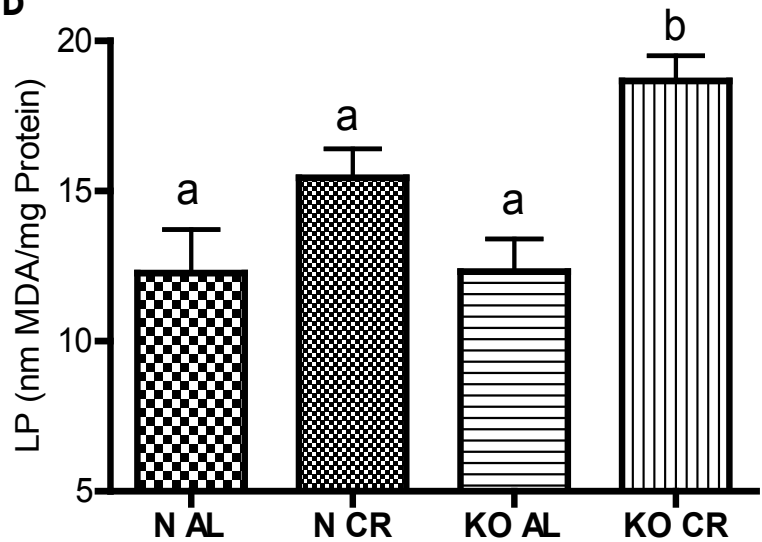

Figure 3. Renal antioxidant enzyme activity (a) catalase, (b) ) Cu/ZnSOD, (c) Gpx 1, and (d) lipid peroxidation (LP) in female GHR-KO $(\mathrm{KO})$ and normal $(\mathrm{N})$ mice subjected to caloric restriction (CR) or fed ad libitum (AL). Data reported as mean $\pm \mathrm{SE}$. Groups that do not share a common superscript are significantly different $(p<0.05)$.

that Ames dwarf mice have extended life as compared to wild type animals (Brown-Borg et al 1996). Furthermore, CR extended the lifespan of long-lived Ames dwarves (Bartke et al., 2001 ). Studies on murine models suggested that CR significantly increases the activity of several antioxidant enzymes including catalase, superoxide dismutase, and glutathione peroxidase (Koizumi et al 1987, Rao et al 1990, Gomi et al 1998). Ames dwarf mice, having mutations for less pituitary secretions, exhibited enhanced activity of antioxidant enzymes in the liver and kidney than age matched normal controls which showed that increased activity of these enzymes have protective effect against oxidative damage and consequently increases the life span (Brown-Borg et al., 1999, 2000 and Hauck and Bartke, 2000). As mentioned earlier, CR did not increase the overall, median or average life span of GHRKO animals and it is suggested that somatotropic signaling is important in mediating the effects of CR on life span of animals (Bonkowski et al., 2006). In the current study we sought to elucidate the effects of $C R$ on the activity of antioxidant enzymes in the liver and kidney of these GHRKO animals.

Catalase is an antioxidant enzyme found in all aerobic cells that catalyzes the decomposition of hydrogen peroxide to oxy- gen and water. A study by Perez et al. (2009) has shown that transgenic mice overexpressing SOD1, catalase or and manganese superoxide dismutase (MnSOD) did not extend the life span of animals. In our study, GHRKO animals had higher catalase activity in the liver and $C R$ reduced it in both normal and GHRKO animals (Figure 2A). In contrast, in the kidney catalase activity was lower in GHRKO animals with no effect of diet (Figure $3 \mathrm{~A}$ ). these tissue specific differences might suggest different mechanisms of somatotropic signaling and $C R$ on antioxidant capacity. It also questions the notion that catalase mediates the longevity induced by $C R$.

CuZnSOD (SOD) is the major superoxide dismutase isozyme found in cells. Mice deficient in SOD1 showed $30 \%$ reduction in life span (Elchuri et al., 2005; Perez et al., 2009) However, overexpression of SOD did not affect the life span in mice (Huang et al, 2000). SOD1 in the kidney was shown to be protective against oxidative damage. Injury to diabetic rodent kidneys has been reduced by overexpression of the antioxidant SOD (Craven et al, 2001). Moreover, SOD activity was found to be reduced in diabetic nephropathy (Fujita et al, 2009). Nishikawa et al demonstrated the importance of ROS generation in mediating hyperglycemia-induced cellular damage (Nishikawa 
et al, 2000). In the current study, GHRKO animals had higher SODI activity in the liver and $C R$ reduced it in these animals (Figure 2B). In the kidney, GHRKO animals have significantly lower SOD activity with no effect of diet (Figure 3B). It was shown that increasing SOD concentrations in mammalian cells produce a paradoxical pro-oxidant action by which protection against oxidative stress is lost and injury was even exacerbated, with increased lipid peroxidation (reviewed in McCord and Edeas, 2005). Reduced SOD activity in GHRKO kidney might provide a balanced protection against ROS injury. These results also suggest different effects of GH/IGF-1 and CR on antioxidant capacity.

Studies in Gpxl-deficient mice have revealed many useful rules of Gpxl in regulating acute oxidative stress. Gpx 1 knockout mice appear to be phenotypically normal, However they are highly susceptible to injury induced by paraquat (a superoxide generator), cerebral ischemia-reperfusion (stroke), and cold-induced head trauma (De Haan et al, 2003). However, Gpx 1 and SOD2 deficiency in mice does not induce lifespan reduction despite increased oxidative damage (Zhang et al, 2009). In our study, in contrast to catalase and SOD 1, Gpx 1 activity was lower in GHRKO liver but higher in the kidney (Figures $2 \mathrm{C}$ and $3 \mathrm{C}$ ). It was previously shown that GHRKO and Ames dwarf mice have less severe glomerulonephritis as compared to normal animals (lkeno et al, 2003). GHRKO kidneys appear to be protected against oxidative damage as indicated by increased Gpx 1 activity. However, CR did increase Gpx 1 activity in normal animals but decreased it in GHRKOs. This adds to the finding that CR did not further increase the lifespan of GHRKO animals (Bonkowski et al, 2006).

This study identified a high level of redundancy for antioxidant enzymes and oxidative stress as mediators of improved longevity in CR and GHRKO animals.

\section{Acknowledgements}

The author would like to thank Dr. Andrzej Bartke for allowing this work to be conducted in his laboratory and for his valuable thoughts and discussions.

\section{References}

Aebi HE (1974) Catalase. In: Bergmeyer HU, ed. Methods in Enzymatic Analysis. New York: Academic Press, 2:673-686.

Bartke (1999) Antioxidative mechanisms and plasma growth hormone levels. Endocrine 11(1): 41-48.

Bartke A, JC Wright, J Mattison, DK Ingram, RA Miller and GS Roth (2001) Longevity: Extending the lifespan of long-lived mice. Nature 414: 412.

Bonkowski MS, JS Rocha, MM Masternak, KA Al Regaiey and A Bartke (2006) Targeted disruption of growth hormone receptor interferes with the beneficial actions of calorie restriction. Proc Natl Acad Sci U S A 103: 7901-7905.

Brown-Borg HM, KE Borg, CJ Meliska and A Bartke (1996) Dwarf mice and the ageing process. Nature 384: 33.

Colman RJ, RM Anderson, SC Johnson, EK Kastman , KJ Kosmatka, TM Beasley, DB Allison, C Cruzen, HA Simmons, JW Kemnitz and $R$ Weindruch (2009) Caloric restriction delays disease onset and mortality in rhesus monkeys. Science 325(5937): 201-4.

Craven PA, MF Melhem, SL Phillips, and FR DeRubertis (2001) Overexpression of $\mathrm{Cu} 2+/ \mathrm{Zn} 2+$ superoxide dismutase protects against early diabetic glomerular injury in transgenic mice. Diabetes 50 : $2114-2125$.

De Haan JB, PJ Crack, N Flentjar, RC lannello, PJ Hertzog, and I Kola (2003) An imbalance in antioxidant defense affects cellular function: the pathophysiological consequences of a reduction in antioxidant defense in the glutathione peroxidase-1 (Gpxl) knockout mouse. Redox Rep 8: 69-79.

Elchuri S, TD Oberley, W Qi, RS Eisenstein, L J Roberts, H Van Remmen, CJ Epstein and T-T Huang (2005) CuZnSOD deficiency leads to persistent and widespread oxidative damage and hepatocarcinogenesis later in life. Oncogene. 24:367-80.

Flurkey K, J Papaconstantinou, R Miller, D Harrison (2001) Lifespan extension and delayed immune and aging in mutant mice with defects in growth hormone production. Proc Natl Acad Sci USA 98: 67366741.

Fontana L, DT Villareal, EP Weiss, SB Racette, K Steger-May, S Klein, JO Holloszy, and the Washington University School of Medicine CALERIE Group (2007) Calorie restriction or exercise: effects on coronary heart disease risk factors. A randomized, controlled trial. Am J Physiol Endocrinol Metab 293: E197-E202.

Fujita H, Fujishima H, Chida S, Takahashi K, Qi Z, Kanetsuna Y, Breyer MD, Harris RC, Yamada Y, Takahashi T (2009) Reduction of renal superoxide dismutase in the progressive diabetic nephropathy. J Am Soc Nephrol. 20(6): 1303-13.

Gomi F, Matsuo M. Effects of aging and food restriction on the antioxidant enzyme activity of rat livers. J Gerontol. 1998;53:B161-7.

Hashimoto M, MS Hossain, H Yamasaki, K Yazawa and S Masumura (1999) Effects of eicosapentaenoic acid and docosahexaenoic acid on plasma membrane fluidity of aortic endothelial cells. Lipids 34: 1297-1304.

Hauck S and A Bartke (2000) Effects of growth hormone on hypothalamic catalase and $\mathrm{Cu} / \mathrm{Zn}$ superoxide dismutase. Free Rad Biol Med 28: 970-978.

Hauck SJ, JM Aaron, C Wright, JJ Kopchick, A Bartke (2002) Antioxidant enzymes, free-radical damage, and response to paraquat in liver and kidney of long-living growth hormone receptor/binding protein gene-disrupted mice. Horm Metab Res 34(9): 481-6.

Heilbronn LK, L de Jonge, MI Frisard, JP DeLany, DEL Meyer, J Rood, T Nguyen, CK Martin, J Volaufova, MM Most, FL Greenway, SR Smith, DA Williamson, WA Deutsch, and E Ravussin Pennington CALERIE team (2006) Effect of 6-mo. calorie restriction on biomarkers of longevity, metabolic adaptation and oxidative stress in overweight subjects. JAMA 295(13): 1539-1548.

Huang TT, EJ Carlson, AM Gillespie, Y Shi, CJ Epstein (2000) Ubiquitous overexpression of CuZn superoxide dismutase does not extend life span in mice. J Gerontol A Biol Sci Med Sci 55:B5-9.

Ikeno Y, RT Bronson, GB Hubbard, S Lee, A Bartke (2003) The delayed occurrence of fatal neoplastic diseases in Ames dwarf mice: correlation to the extended longevity. J Gerontol A Biol Sci Med Sci 58:291-296.

Kenyon C, J Chang, E Gensch, A Rudner and R Tabtiang (1993) A C. elegans mutant that lives twice as long as wild type. Nature 366: $461-464$

Koizumi A, R Weindruch and RL Walford (1987) Influences of dietary restriction and age on liver enzyme activities and lipid peroxidation in mice. J Nutr 117:361-367.

Lakowski B and S Hekimi (1998) The genetics of caloric restriction in Caenorhabditis elegans. Proc. Natl. Acad. Sci. USA 95: 13091 13096.

Larson-Meyer DE, LK Heilbronn, LM Redman, BR Newcomer, MI Frisard, 
S Anton, SR Smith, AA Maplstat, E Ravussin, and Pennington CALERIE Team (2006) Effect of Calorie Restriction With or Without Exercise on Insulin Sensitivity, $\beta$-Cell Function, Fat Cell Size, and Ectopic Lipid in Overweight Subjects. Diabetes Care 29(6): 1337-1344.

Martin CK, SD Anton, H Han, E York-Crowe, LM Redman, E Ravussin, DA Williamson, and for the Pennington CALERIE Team (2007) Examination of Cognitive Function During Six Months of Calorie Restriction: Results of a Randomized Controlled Trial. Rejuvenation Research 10(2): 179-190.

Masoro EJ (1998) Hormesis and the antiaging action of dietary restriction. Exp Ger 33: 61-66.

Mattison JA, C Wright, RT Bronson, GS Roth, DK Ingram and A Bartke (2000) Studies of aging in Ames dwarf mice: Effects of caloric restriction. J Amer Aging Assoc (23): 9-16.Amer. Aging Assoc., Vol. 23, 9-16, 2000

McCord J.M and M.A. Edeas (2005) SOD, oxidative stress and human pathologies: a brief history and a future vision. Biomed Pharmacother 59 : 139-1 42.

Melov S, J Ravenscroft, S Malik, MS Gill, DW Walker, PE Clayton, DC Wallace, B Malfroy, SR Doctrow and GJ Lithgow (2000) Extension of life-span with superoxide dismutase/catalase mimetics. Science 289(5484): 1567-1569.

Nishikawa T, D Edelstein, XL Du, S Yamagishi, T Matsumura, Y Kaneda, MA Yorek, D Beebe, PJ Oates, HP Hammes, I Giardino and M Brownlee (2000) Normalizing mitochondrial superoxide production blocks three pathways of hyperglycaemic damage. Nature 404:787-790

Orr WC \& RS Sohal (1994) Extension of life-span by overexpression of superoxide dismutase and catalase in Drosophila melanogaster. Science 263: $1128-1130$.

Parkes TL, JE Andrew, D Dickinson, AJ Hilliker, JP Phillips and GL Boulianne (1998) Extension of Drosophila lifespan by overexpression of human SOD1 in motorneurons. Nat Genet 19: 171-174.

Pérez VI, Bokov A, Van Remmen H, Mele J, Ran Q, lkeno Y, Richardson A. (2009) Is the oxidative stress theory of aging dead? Biochim Biophys Acta.; 1790(10):1005-14.

Pérez VI, H Van Remmen, A Bokov, CJ Epstein, J Viig and A Richardson (2009) The overexpression of major antioxidant enzymes does not extend the lifespan of mice. Aging Cell 8: 73-75

Rao G, E Xia, MJ Nadakavukaren and A Richardson (1990) Effect of dietary restriction on the age-dependent changes in the expression of antioxidant enzymes in rat liver. J Nutr 120:602-609.

Redman LM, LK Heilbronn, CK Martin, A Alfonso, SR Smith, and E Ravussin for the Pennington CALERIE Team (2007) Effect of Calorie Restriction with or without Exercise on Body Composition and Fat Distribution. J Clin Endocrinol Metab 92(3): 865-872.

Schriner SE and NJ Linford (2006) Extension of mouse lifespan by overexpression of catalase. Age (Dordr) 28(2): 209-218.

Sohal RS, R Weindruch (1996) Oxidative stress, caloric restriction, and aging. Science 273(5271): 59-63.

Sun Y and LW Oberley, (1994) Suitability of copper chloride as a reaction terminator for superoxide dismutase activity assay. Clin Chim Acta 226:101-103.

Tappel AL (1978) Glutathione peroxidase and hydroperoxides. In: Fleisher S, Packer L, eds. Methods in Enzymology. New York: Academic Press 52:506-513.

Tatar M, A Bartke A and A Antebi (2003) The endocrine regulation of aging by insulin-like signals. Science 299:1346-135.

Wang ZQ, ZE Floyd, J Qin , X Liu , Y Yu , XH Zhang , JD Wagner , WT Cefalu (2009) Modulation of skeletal muscle insulin signaling with chronic caloric restriction in cynomolgus monkeys. Diabetes 58(7): 1488-98.

Weindruch R and RS Sohal (1997) Seminars in medicine of the Beth Israel Deaconess Medical Center. Caloric intake and aging. N Engl J Med 337: 986-994.

Witte AV, M Fobker , R Gellner , S Knecht, A Flöel (2009) Caloric restriction improves memory in elderly humans. PNAS 106 (4): 1255 60.

Zhang Y, Y Ikeno, W Qi, A Chaudhuri, Y Li, A Bokov, SR Thorpe, JW Baynes, C Epstein, A Richardson, H Van Remmen (2009) Mice Deficient in Both Mn Superoxide Dismutase and Glutathione Peroxidase-1 Have Increased Oxidative Damage and a Greater Incidence of Pathology but No Reduction in Longevity. J Gerontol A Biol Sci Med Sci 64 (12): 1212-1220. 